\title{
THE MATRIX MODEL FOR HYPERGEOMETRIC HURWITZ NUMBERS
}

\author{
JAN AMBJøRN ${ }^{\dagger}$ AND LEONID CHEKHOV*
}

\begin{abstract}
We present the multi-matrix models that are the generating functions for branched covers of the complex projective line ramified over $n$ fixed points $z_{i}, i=1, \ldots, n$, (generalized Grotendieck's dessins d'enfants) of fixed genus, degree, and the ramification profiles at two points, $z_{1}$ and $z_{n}$. We take a sum over all possible ramifications at other $n-2$ points with the fixed length of the profile at $z_{2}$ and with the fixed total length of profiles at the remaining $n-3$ points. All these models belong to a class of hypergeometric Hurwitz models thus being tau functions of the Kadomtsev-Petviashvili (KP) hierarchy. In the case described above, we can present the obtained model as a chain of matrices with a (nonstandard) nearest-neighbor interaction of the type $\operatorname{tr} M_{i} M_{i+1}^{-1}$. We describe the technique for evaluating spectral curves of such models, which opens the possibility of applying the topological recursion for developing $1 / N^{2}$-expansions of these model. These spectral curves turn out to be of an algebraic type.
\end{abstract}

\section{INTRODUCTION}

In general, Hurwitz numbers pertain to combinatorial classes of ramified mappings $f$ : $\mathbb{C} P^{1} \rightarrow \Sigma_{g}$ of the complex projective line onto a Riemann surface of genus $g$. Commonly, single or double Hurwitz numbers correspond to the cases in which ramification profiles (defined by the corresponding Young tableauxes $\lambda$ or $\lambda$ and $\mu$ ) are respectively determined at one $(\infty)$ or two $(\infty$ and 1$)$ distinct points whereas we assume the existence of $m$ other distinct ramification points with only simple ramifications.

Generating functions for Hurwitz numbers have been considered for long in mathematical physics. Notably, Okounkov and Pandharipande [27] had shown that the exponential of the generating function for double Hurwitz numbers is a tau-function of the Kadomtsev-Petviashvili (KP) hierarchy. The same result was obtained by A. Yu. Orlov and Shcherbin [28], 29] using the Schur function technique and, in a more general setting, by Goulden and Jackson [18] using Plucker relations.

Orlov and Shcherbin [28] also addressed the case of the generating function for the case of Grothendieck dessins d'enfants where we have only three ramification points with multiple ramifications and the ramification profile is fixed at one or two of these points. In this case, they have also concluded that the exponentials of the corresponding generating functions have to be tau functions of the KP hierarchy. Actually, the results of [28] describe a wider class of generating functions for hypergeometric Hurwitz numbers (this term was coined there) in which we have a fixed number $n$ of ramification points in $\mathbb{C} P^{1}$ assuming that we fix profiles at two of these points and take a sum over profiles at all other points with weights being proportional to the lengths of the remaining $n-2$ profiles. Recently, Harnad and Orlov [21] showed that all these generating functions are in turn tau functions of the KP hierarchy.

The interest to Hurwitz numbers corresponding to Belyi pairs was revived by Zograf [30] (see also [23]) who provided recursion relations for the generating function of Grothendieck's dessins d'enfants enumerating the Belyi pairs $(C, f)$, where $C$ is a smooth algebraic curve and

${ }^{\dagger}$ Niels Bohr Institute, Copenhagen University, Denmark, and IMAPP, Radboud University, Nijmengen, The Netherlands. Email: ambjorn@nbi.dk.

*Steklov Mathematical Institute and Laboratoire Poncelet, Moscow, Russia; Center for Quantum Geometry of Moduli Spaces, Århus University, Denmark. Email: chekhov@mi.ras.ru. 
$f$ a meromorphic function $f: C \rightarrow \mathbb{C} P^{1}$ ramified only over the points $0,1, \infty \in \mathbb{C} P^{1}$. In [3], we proposed the matrix-model description of Belyi pairs, clean Belyi morphisms, and two-profile Belyi pairs thus showing that all these cases fall into the category of KP tau functions. Then the multi-matrix-model representation for the hypergeometric Hurwitz numbers was constructed in [2] but with a complicated interaction between matrices in the chain. In the present note, we propose a more standard description of hypergeometric Hurwitz numbers in the case where we fix profiles at two ramification points, fix the length of the profile at the third point, and fix the total length of profiles at other $n-3$ points.

We recall some mathematical results relating Belyi pairs to Galois groups.

Theorem 1.1. (Belyi, [5]) A smooth complex algebraic curve $C$ is defined over the field of algebraic numbers $\overline{\mathbb{Q}}$ if and only if we have a nonconstant meromorphic function $f$ on $C(f$ : $\left.C \rightarrow \mathbb{C} P^{1}\right)$ ramified only over the points $0,1, \infty \in \mathbb{C} P^{1}$.

For a Belyi pair $(C, f)$ let $g$ be the genus of $C$ and $d$ the degree of $f$. If we take the preimage $f^{-1}([0,1]) \subset C$ of the real line segment $[0,1] \in \mathbb{C} P^{1}$ we obtain a connected bipartite fat graph with $d$ edges with vertices being preimages of 0 and 1 and such that the cyclic ordering of edges entering a vertex comes from the orientation of the curve $C$. This led Grothendieck to formulating the following lemma.

Lemma 1.2. (Grothendieck, [19]) There is a one-to-one correspondence between the isomorphism classes of Belyi pairs and connected bipartite fat graphs.

A Grothendieck dessin d'enfant is therefore a connected bipartite fat graph representing a Belyi pair. It is well known that we can naturally extend the dessin $f^{-1}([0,1]) \subset C$ corresponding to a Belyi pair $(C, f)$ to a bipartite triangulation of the curve $C$. For this, we cut the complex plane along the (real) line containing $0,1, \infty$ coloring upper half plane white and lower half plane gray. This defines the partition of $C$ into white and grey triangles such that white triangles has common edges only with grey triangles. We then consider a dual graph in which edges are of three types.

In this paper, we consider generalized Belyi pairs, which are mappings $\left(f: C \rightarrow \mathbb{C} P^{1}\right)$ with possible ramifications over $n$ fixed points $z_{i} \in \mathbb{C} P^{1}, i=1, \ldots, n$. We then have the splitting of the curve $C$ into bipartite $n$-gons with $n$ colored edges (the corresponding fat graphs are then coverings of the basic graph depicted in Fig. 1 for $n=5$ ): the type of an edge depend on which of $n$ segments of $\mathbb{R} P^{1-f^{-1}}\left(\left[\infty_{-}, z_{2}\right]\right) \subset C, f^{-1}\left(\left[z_{2}, z_{3}\right]\right) \subset C, \ldots, f^{-1}\left(\left[z_{n-1}, z_{n}\right]\right) \subset C$, $f^{-1}\left(\left[z_{n}, \infty_{+}\right]\right) \subset C$-it intersects (we identify $z_{1}$ with the infinity point and let $\infty_{ \pm}$indicate the directions of approaching this point along the real axis in $\left.\mathbb{C} P^{1}\right)$. Each face of the dual partition then contains a preimage of exactly one of the points $z_{1}, \ldots, z_{n}$, so these faces are of $n$ sorts (bordered by solid, dotted, or dashed lines in the figure). We call such a graph a generalized Belyi fat graph.

The type of ramification at infinity is determined by the set of solid-line-bounded faces of a generalized Belyi fat graph: the order of branching is $r$ for a $2 r$-gon, so we introduce the generating function that distinguishes between different types of branching at infinity, or $z_{1}$. Moreover, we also distinguish between different types of ramifications at the $n$th point (the point $(3+\sqrt{5}) / 2$ in Fig. 10. This situation is often called a two-profile generating function for Hurwitz numbers because we fix two ramification patterns at two distinct branching points; each such pattern can be represented by a Young tableaux. We let $k_{i}$ denote the numbers of respective cycles (pre-images of the points $z_{i}$ on the Riemann surface $C$ ) and let $k_{1}^{(r)}$ and $k_{n}^{(r)}$ denote the numbers of cycles of length $2 r$ centered at pre-images of the respective points $z_{1}$ and $z_{n}$ in a generalized Belyi fat graph. 


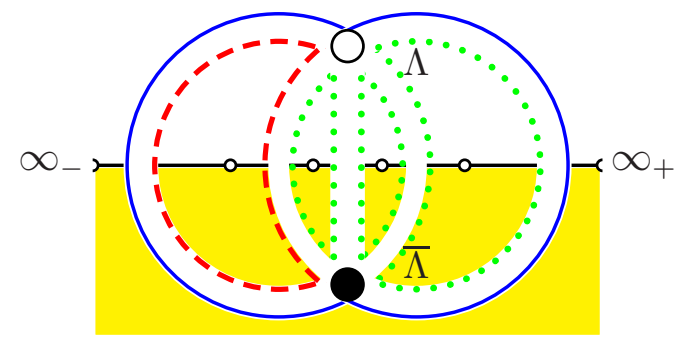

FiguRE 1. The generalized Belyi graph $\Gamma_{1}$ corresponding to possible ramifications at $n=5$ points (commonly taken to be $\infty,-(1+\sqrt{5}) / 2,0,1$, and $(3+\sqrt{5}) / 2$; we denote them by small white circles). This graph describes the generalized Belyi pair $\left(\mathbb{C} P^{1}\right.$, id); $\infty_{ \pm}$indicate directions of approaching the infinite point in $\mathbb{C} P^{1}$. The symbols $\Lambda$ and $\bar{\Lambda}$ indicate the insertions of the external field in the matrix-model formalism of Sec. 2 . For example, this graph contributes the term $N^{2} \gamma_{1} \gamma_{2} \gamma_{3}^{2} t_{1} \operatorname{tr}(\Lambda \bar{\Lambda})$.

As was shown in [2] and [21], the exponential of the generating function

$$
\mathcal{F}\left[\left\{t_{m}\right\},\left\{\mathfrak{t}_{r}\right\}, \gamma_{2}, \ldots, \gamma_{n-1} ; N\right]=\sum_{\Gamma} \frac{1}{|\operatorname{Aut} \Gamma|} N^{2-2 g} \prod_{r=1}^{\infty} t_{r}^{k_{1}^{(r)}} \prod_{s=1}^{\infty} \mathfrak{t}_{s}^{k_{n}^{(s)}} \prod_{j=2}^{n-1} \gamma_{j}^{k_{j}}
$$

is a tau function of the KP hierarchy in times $t$ or $\mathfrak{t}$. Although a matrix-model description of this generating function was proposed in the above papers, the possibility of solving it in topological recursion terms (see [16], [10], [11]) remained obscure. We are going to construct a matrix model describing a subclass of generating functions (1.1) with $\gamma_{3}=\gamma_{4}=\cdots=\gamma_{n-1}$ leaving $\gamma_{2}$ arbitrary.

Our goal in the present paper is therefore to construct and solve a matrix model whose free energy is the generating function

$$
\mathcal{F}\left[\left\{t_{m}\right\},\left\{\mathfrak{t}_{r}\right\}, \gamma_{2}, \gamma_{3} ; N\right]=\sum_{\Gamma} \frac{1}{|\operatorname{Aut} \Gamma|} N^{2-2 g} \prod_{r=1}^{\infty} t_{r}^{k_{1}^{(r)}} \prod_{s=1}^{\infty} \mathfrak{t}_{s}^{k_{n}^{(s)}} \gamma_{2}^{k_{2}} \gamma_{3}^{k_{3}+\cdots+k_{n-1}},
$$

where $N, \gamma_{2}, \gamma_{3}, t_{r}$, and $\mathfrak{t}_{r}$ are formal independent parameters and the sum ranges all (connected) generalized Belyi fat graphs. Below we are dealing with a matrix model with an external matrix field $\Lambda=\operatorname{diag}\left(\lambda_{1}, \ldots, \lambda_{\gamma_{3} N}\right)$, the corresponding times are

$$
\mathfrak{t}_{r}=\operatorname{tr}\left[(\Lambda \bar{\Lambda})^{r}\right]
$$

Sometimes factors $\gamma_{1}^{k_{1}}$ and $\gamma_{n}^{k_{n}}$ are added but they can always be absorbed into the times $t_{r}$ and $\mathfrak{t}_{r}$ by scaling $t_{r} \rightarrow \gamma_{1} t_{r}$ and $\mathfrak{t}_{r} \rightarrow \gamma_{n} \mathfrak{t}_{r}$ for all $r$.

The structure of the paper is as follows. In Sec. 2, we show that generating function (1.2) is the free energy of a special multi-matrix model represented as a chain of matrices with somewhat nonstandard interaction terms $\operatorname{tr} M_{i} M_{i+1}^{-1}$. We express this model as an integral over eigenvalues of these matrices in a form similar to that of the standard generalized Kontsevich model (GKM) [24]. We adapt the technique of Eynard and Prats Ferrer [17] to evaluating spectral curves for chains of matrices with these nonstandard interaction terms in Sec. 4. Although we derive the spectral curve only in the first nontrivial case $n=4$ (the case of one intermediate field), our technique can be straightforwardly generalized to all higher $n$, which will be done in a separate publication. We conclude with the discussion of our results.

Throughout the entire text we disregard all multipliers not depending on external fields and times $t_{r}$; all equalities in the paper must be therefore understood modulo such factors. 


\section{THE MODEL}

In order to take into account the profile at the infinity point, we first contract all solid cycles (centered at pre-images of $\infty$ ) assigning the time $t_{r}$ to every contracted cycle of length $2 r$.

The new interaction vertices arise from the thus contracted solid cycles. For example, for a cycle of length four, we obtain the correspondence

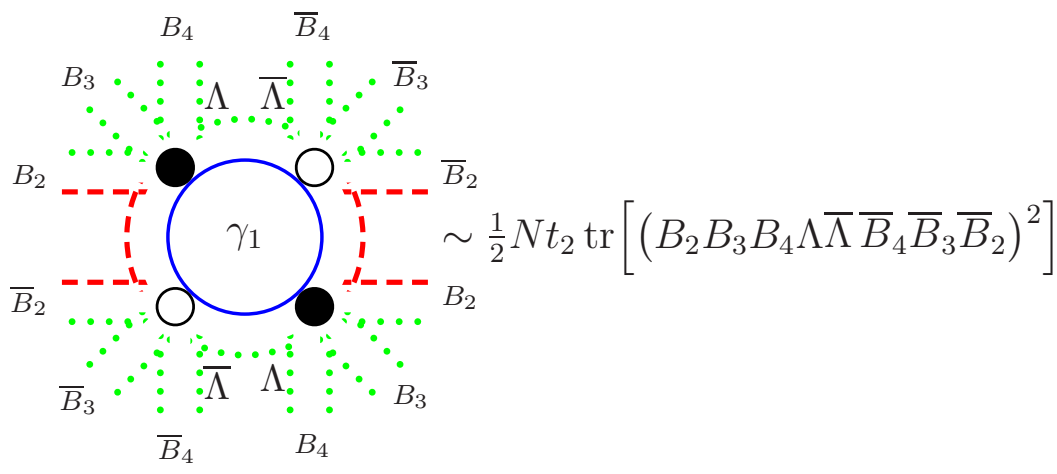

where the factor $1 / 2$ takes into account the symmetry of the four-cycle.

The matrix-valued fields $B_{i}, i=2, \ldots, n-1$, are general complex-valued matrices such that $B_{2}$ is a rectangular matrix of the size $\gamma_{2} N \times \gamma_{3} N$ and we always assume that

$$
\gamma_{2}>\gamma_{3}
$$

and all other matrices $B_{3}, \ldots, B_{n-1}$ are square matrices of the size $\gamma_{3} N \times \gamma_{3} N$.

The matrix-model integral whose free energy is the generating function (1.2) reads

$$
\int D B_{2} \cdots D B_{n-1} e^{N \sum_{r=1}^{\infty} \frac{t_{r}}{r} \operatorname{tr}\left[\left(B_{2} \cdots B_{n-1} \Lambda \bar{\Lambda} \bar{B}_{n-1} \cdots \bar{B}_{2}\right)^{r}\right]-\sum_{j=2}^{n-1} N \operatorname{tr}\left(B_{j} \bar{B}_{j}\right)}
$$

We next perform the variable changing

$$
\begin{aligned}
& \mathfrak{B}_{2}=B_{2} B_{3} \cdots B_{n-1} \\
& \mathfrak{B}_{3}=B_{3} \cdots B_{n-1} \\
& \vdots \\
& \mathfrak{B}_{n-1}=B_{n-1}
\end{aligned}
$$

and assume that all matrices $\mathfrak{B}_{3}, \ldots, \mathfrak{B}_{n-1}$ are invertible (the matrix $\mathfrak{B}_{2}$ remains rectangular). With accounting for the Jacobian of transformation (2.2), the integral (2.1) becomes

$$
\begin{aligned}
& \int D \mathfrak{B}_{2} \cdots D \mathfrak{B}_{n-1} \exp \left\{-\gamma_{2} N \operatorname{tr} \log \left(\mathfrak{B}_{3} \overline{\mathfrak{B}}_{3}\right)-\sum_{j=4}^{n-1} \gamma_{3} N \operatorname{tr} \log \left(\mathfrak{B}_{j} \overline{\mathfrak{B}}_{j}\right)\right. \\
& +\sum_{r=1}^{\infty} N \frac{t_{r}}{r} \operatorname{tr}\left[\left(\mathfrak{B}_{2}|\Lambda|^{2} \overline{\mathfrak{B}}_{2}\right)^{r}\right]-N \operatorname{tr}\left[\mathfrak{B}_{2} \mathfrak{B}_{3}^{-1} \overline{\mathfrak{B}}_{3}^{-1} \overline{\mathfrak{B}}_{2}\right] \\
& \left.-N \operatorname{tr}\left[\mathfrak{B}_{3} \mathfrak{B}_{4}^{-1} \overline{\mathfrak{B}}_{4}^{-1} \overline{\mathfrak{B}}_{3}\right]-\cdots-N \operatorname{tr}\left[\mathfrak{B}_{n-2} \mathfrak{B}_{n-1}^{-1} \overline{\mathfrak{B}}_{n-1}^{-1} \overline{\mathfrak{B}}_{n-2}\right]-N \operatorname{tr}\left[\mathfrak{B}_{n-1} \overline{\mathfrak{B}}_{n-1}\right]\right\} .
\end{aligned}
$$

Here it becomes clear why we demand all matrices except $\mathfrak{B}_{2}$ to be quadratic: we must be able to invert them in order to write the corresponding generating function as a free energy of a chain of Hermitian matrices, as we demonstrate below. 
We now recall [4] that we can write an integral over general complex matrices $\mathfrak{B}_{i}$ in terms of positive definite Hermitian matrices $X_{i}$ upon the variable changing

$$
X_{i}:=\overline{\mathfrak{B}}_{i} \mathfrak{B}_{i}, \quad i=2, \ldots, n-1 .
$$

All the matrices $X_{i}(i=2, \ldots, n-1)$ are of the same size $\gamma_{3} N \times \gamma_{3} N$. Changing the integration measure for rectangular complex matrices introduces just a simple logarithmic term (see, e.g., [3]) and the resulting integral becomes

$$
\begin{aligned}
& \int D X_{2 \geq 0} \cdots D X_{n-1 \geq 0} \exp \left\{N \sum_{r=1}^{\infty} \frac{t_{r}}{r} \operatorname{tr}\left[\left(X_{2}|\Lambda|^{2}\right)^{r}\right]-N \operatorname{tr}\left(X_{2} X_{3}^{-1}\right)-\cdots-N \operatorname{tr}\left(X_{n-2} X_{n-1}^{-1}\right)\right. \\
& \left.-N \operatorname{tr} X_{n-1}+\left(\gamma_{2}-\gamma_{3}\right) N \operatorname{tr} \log X_{2}-\gamma_{2} N \operatorname{tr} \log X_{3}-\gamma_{3} N \operatorname{tr} \log \left(X_{4} \cdots X_{n-1}\right)\right\} .
\end{aligned}
$$

The logarithmic term in $X_{2}$ stabilizes the equilibrium distribution of eigenvalues of this matrix in the domain of positive real numbers; in the case where $\gamma_{2}=\gamma_{3}$, we lose this term and must use the technique of matrix models with hard walls (for a review, see, e.g., [8]).

Making a scaling $X_{i} \rightarrow X_{i}|\Lambda|^{-2}$ for all the integration variables, we reduce (2.5) to a more familiar form of an integral over a chain of matrices,

$$
\begin{aligned}
\int D X_{2 \geq 0} \cdots D X_{n-1 \geq 0} \exp \left\{N \sum_{r=1}^{\infty} \frac{t_{r}}{r} \operatorname{tr}\left(X_{2}^{r}\right)-N \operatorname{tr}\left(X_{2} X_{3}^{-1}\right)-\cdots-N \operatorname{tr}\left(X_{n-2} X_{n-1}^{-1}\right)\right. \\
\left.-N \operatorname{tr}\left(X_{n-1}|\Lambda|^{-2}\right)+\left(\gamma_{2}-\gamma_{3}\right) N \operatorname{tr} \log X_{2}-\gamma_{2} N \operatorname{tr} \log X_{3}-\gamma_{3} N \operatorname{tr} \log \left(X_{4} \cdots X_{n-1}\right)\right\}
\end{aligned}
$$

We use this expression when deriving the spectral curve equation in the next section. Now we proceed further expressing integral (2.6) in terms of eigenvalues $x_{i}^{(k)}$ of the matrices $X_{k}$, $k=2, \ldots, n-1$.

We apply the Mehta-Itzykson-Zuber integration formula to every term in the chain of matrices in (2.6). Taking into account that, for instance, the integral over the unitary group for the term $e^{-N \operatorname{tr} X_{k} X_{k+1}^{-1}}$ gives

$$
\int D U e^{-N \sum_{i, j=1}^{\gamma_{3} N} U_{i j} x_{i}^{(k)} U_{i j}^{*}\left[x_{j}^{(k+1)}\right]^{-1}}=\frac{\operatorname{det}_{i, j}\left[e^{-N x_{i}^{(k)} / x_{j}^{(k+1)}}\right]}{\Delta\left(x^{(k)}\right) \Delta\left(1 / x^{(k+1)}\right)}
$$

and that $1 / \Delta\left(1 / x^{(k+1)}\right)=\prod_{i=1}^{\gamma_{3} N}\left[x_{i}^{(k+1)}\right]^{\gamma_{3} N-1} / \Delta\left(x^{(k+1)}\right)$ we eventually write the expression in terms of eigenvalues of the matrices $X_{k}$ :

$$
\begin{aligned}
& \int_{0}^{\infty} \prod_{i=1}^{\gamma_{3} N} d x_{i}^{(2)} \frac{\Delta\left(x^{(2)}\right)}{\Delta\left(|\Lambda|^{-2}\right)} \prod_{k=3}^{n-1}\left(\prod_{i=1}^{\gamma_{3} N} \frac{d x_{i}^{(k)}}{x_{i}^{(k)}}\right) \times \\
& \quad \times \prod_{i=1}^{\gamma_{3} N}\left[\left(x_{i}^{(2)} / x_{i}^{(3)}\right)^{\left(\gamma_{2}-\gamma_{3}\right) N} e^{N \sum_{r=1}^{\infty} \frac{t_{r}}{r}\left(x_{i}^{(2)}\right)^{r}-N x_{i}^{(2)} / x_{i}^{(3)}-\cdots-N x_{i}^{(n-2)} / x_{i}^{(n-1)}-N x_{i}^{(n-1)}|\Lambda|_{i}^{-2}}\right]
\end{aligned}
$$

Finally, if we introduce logarithmic quantities

$$
\varphi_{i}^{(r)}=\log x_{i}^{(r)}, \quad r=3, \ldots, n-1,
$$

we can rewrite integral (2.7) in a more transparent form resembling that of the Today chain:

$$
\begin{aligned}
& \int_{0}^{\infty} \prod_{i=1}^{\gamma_{3} N} d x_{i}^{(2)} \frac{\Delta\left(x^{(2)}\right)}{\Delta\left(|\Lambda|^{-2}\right)} \prod_{i=1}^{\gamma_{3} N}\left[\int_{-\infty}^{\infty} \prod_{k=3}^{n-1} d \varphi_{i}^{(k)} \times\right. \\
& \quad \times \exp \left[N \sum_{r=1}^{\infty} \frac{t_{r}}{r}\left(x_{i}^{(2)}\right)^{r}+\left(\gamma_{2}-\gamma_{3}\right) N \log x_{i}^{(2)}-\left(\gamma_{2}-\gamma_{3}\right) N \varphi_{i}^{(3)}\right.
\end{aligned}
$$




$$
\left.\left.-N x_{i}^{(2)} e^{-\varphi_{i}^{(3)}}-N e^{\varphi_{i}^{(3)}-\varphi_{i}^{(4)}}-\cdots-N e^{\varphi_{i}^{(n-2)}-\varphi_{i}^{(n-1)}}-N e^{\varphi_{i}^{(n-1)}}|\Lambda|_{i}^{-2}\right]\right] .
$$

In this form it is clear that all integrals w.r.t. $\varphi_{i}^{(k)}$ are convergent.

\section{The CASE of tWo-Profile Belyi morphism for $n=3$}

We now recall the results of [3] where the case $n=3$ was considered. In this case, we do not have "intermediate" integrations over $\varphi_{i}$ in (2.8) and the partition function is described by the following lemma.

Lemma 3.1. In the case where we allow only three ramification points: 0 , 1 , and $\infty$, the generating function

$$
\mathcal{F}\left[\left\{t_{1}, t_{2}, \ldots\right\},\left\{\mathfrak{t}_{1}, \mathfrak{t}_{2}, \ldots\right\}, \beta ; N\right]=\sum_{\Gamma} \frac{1}{|A u t \Gamma|} N^{2-2 g} \beta^{n_{2}} \prod_{i=1}^{n_{1}} t_{r_{i}} \prod_{k=1}^{n_{3}} \mathfrak{t}_{s_{k}}
$$

of Belyi morphisms in which we fix two sets of ramification profiles: $\left\{t_{r_{1}}, \ldots, t_{r_{n_{1}}}\right\}$ at infinity and $\left\{\mathfrak{t}_{s_{1}}, \ldots, \mathfrak{t}_{s_{n_{3}}}\right\}$ at 1 and we take a sum over profiles at zero, is given by the integral over Hermitian positive definite $(\gamma N \times \gamma N)$-matrix $X$ with the external matrix field $\tilde{\Lambda}:=|\Lambda|^{-2}$ :

$$
\mathcal{Z}[t, \mathfrak{t}]=\prod_{k=1}^{\gamma N}\left|\lambda_{k}\right|^{-2 \beta N} \int_{\gamma N \times \gamma N} D X_{\geq 0} e^{N \operatorname{tr}\left[-X|\Lambda|^{-2}+\sum_{m=1}^{\infty} \frac{t_{m}}{m} X^{m}+(\beta-\gamma) \log X\right]} .
$$

Here $\mathfrak{t}_{s}=\operatorname{tr}\left[(\Lambda \bar{\Lambda})^{s}\right]$.

Integral (3.2) is a GKM integral [24]; after integration over eigenvalues $x_{k}$ of the matrix $X$ it acquires the form of the ratio of two determinants,

$$
\mathcal{Z}[t, \mathfrak{t}]=\prod_{k=1}^{\gamma N}\left|\lambda_{k}\right|^{-2 \beta N} \frac{\left\|\frac{\partial^{k_{1}-1}}{\partial \tilde{\lambda}_{k_{2}}^{k_{1}-1}} f\left(\tilde{\lambda}_{k_{2}}\right)\right\|_{k_{1}, k_{2}=1}^{\gamma N}}{\Delta(\tilde{\lambda})}
$$

where

$$
f(\tilde{\lambda})=\int_{0}^{\infty} x^{N(\beta-\gamma)} e^{-N x \tilde{\lambda}+N} \sum_{m=1}^{\infty} \frac{t_{m}}{m} x^{m} .
$$

Because any GKM integral (in the proper normalization) is a $\tau$-function of the KP hierarchy, and for a model with the logarithmic term in the potential it was demonstrated in [25], we immediately come to the conclusion that the exponential $e^{\mathcal{F}[\{t\},\{t\}, \gamma ; N]}$ of generating function (3.1) modulo the normalization factor $\prod_{k=1}^{\gamma N}\left|\lambda_{k}\right|^{-2 \beta N}$ is a $\tau$-function of the KP hierarchy (that is, it satisfies the bilinear Hirota relations) in times $\mathfrak{t}_{s}$ described in Lemma 3.1 .

\section{Spectral CURVe And topological ReCURsion}

In this section, we propose the method for deriving the spectral curve of model (2.6) adapting the technique of [17] to our case of a nonstandard interaction between matrices in the matrix chain. In the present short paper, we restrict ourselves to a technically more transparent case of the three-matrix model given by the integral

$$
\int D M_{1} D M_{2} D M_{3} e^{N \operatorname{tr}\left[V\left(M_{1}\right)+M_{1} M_{2}^{-1}-\gamma_{2} \log M_{2}+M_{2} M_{3}+U\left(M_{3}\right)\right]},
$$

where the integrations are performed w.r.t. positive-definite Hermitian matrices of size $\gamma_{3} N \times$ $\gamma_{3} N$ and potentials $V(x)$ and $U(x)$ are two Laurent polynomials of the respective positive 
degrees $n$ and $r$ (this consideration can be easily generalized to the case where $V^{\prime}(x)$ and $U^{\prime}(x)$ are two rational functions).

The model (4.1) satisfies [21, [20] equations of the two-dimensional Toda chain hierarchy, not those of the KP hierarchy, but these two classes of models are closely related by Miwatype transformations, so solving the problem of finding the spectral curve in one model can be translated in a standard way to solving the corresponding problem in the other model. Because finding spectral curves for multi-matrix models is technically somehow more transparent than finding spectral curves for models with external matrix fields, we stay with the first choice.

We consider the following variations of the matrix fields $M_{i}$ :

$$
\begin{aligned}
\delta M_{1} & =\frac{1}{x-M_{1}} \xi\left(M_{2}, M_{3}\right), \\
\delta M_{2} & =M_{2} \frac{1}{x-M_{1}} \eta\left(M_{1}, M_{3}\right), \\
\delta M_{3} & =\frac{1}{x-M_{1}} \rho\left(M_{1}, M_{2}\right),
\end{aligned}
$$

where we choose $\xi, \eta$, and $\rho$ to be Laurent polynomials in their arguments. We introduce the standard notation for the leading term of the $1 / N^{2}$-expansion of the one-loop mean of the matrix field $M_{1}$ :

$$
\omega_{1}(x):=\frac{1}{N}\left\langle\operatorname{tr} \frac{1}{x-M_{1}}\right\rangle_{0} .
$$

Here and hereafter, the subscript 0 of a correlation function indicates the contribution of the leading order in $1 / N^{2}$-expansion. A single trace symbol pertains to the whole expression inside the corresponding brackets.

The exact loop equations obtained upon variations (4.2) read

$$
\begin{aligned}
& \frac{1}{N^{2}}\left\langle\operatorname{tr} \frac{1}{x-M_{1}} \operatorname{tr} \frac{1}{x-M_{1}} \xi\left(M_{2}, M_{3}\right)\right\rangle^{\mathrm{c}}+\left[\omega_{1}(x)+V^{\prime}(x)\right]\left\langle\operatorname{tr} \frac{1}{x-M_{1}} \xi\left(M_{2}, M_{3}\right)\right\rangle \\
& \quad+\left\langle\operatorname{tr} \frac{V^{\prime}\left(M_{1}\right)-V^{\prime}(x)}{x-M_{1}} \xi\left(M_{2}, M_{3}\right)\right\rangle+\left\langle\operatorname{tr} M_{2}^{-1} \frac{1}{x-M_{1}} \xi\left(M_{2}, M_{3}\right)\right\rangle=0 \\
& \left\langle\operatorname{tr} \frac{-M_{1}}{x-M_{1}} \eta\left(M_{1}, M_{3}\right) M_{2}^{-1}\right\rangle+\left\langle\operatorname{tr} M_{3} M_{2} \frac{1}{x-M_{1}} \eta\left(M_{1}, M_{3}\right)\right\rangle \\
& \quad+\left(\gamma_{2}-\gamma_{3}\right)\left\langle\operatorname{tr} \frac{1}{x-M_{1}} \eta\left(M_{1}, M_{3}\right)\right\rangle=0 ; \\
& \left\langle\operatorname{tr} M_{2} \frac{1}{x-M_{1}} \rho\left(M_{1}, M_{2}\right)\right\rangle+\left\langle\operatorname{tr} U^{\prime}\left(M_{3}\right) \frac{1}{x-M_{1}} \rho\left(M_{1}, M_{2}\right)\right\rangle=0 .
\end{aligned}
$$

A complete information on the model is encoded in these loop equations; solving them we can develop the topological recursion procedure for evaluating terms in the $1 / N^{2}$-expansion. Our goal in this paper is however more modest: we are only going to derive the spectral curve (this nevertheless ensures all the necessary ingredients of the topological recursion [16], [10], [11, see also [1], which are the spectral curve itself and two meromorphic differentials defined on this curve).

Because we obtain the spectral curve in the large- $N$ limit, we disregard the first term in (4.4), which is of the next order in $1 / N^{2}$. All other terms in all three equations contribute to the leading order.

We next perform several substitutions enabling us to produce the required identities; in all the identities below we keep only leading terms in the large- $N$ limit: 
The first substitution is

$$
\begin{aligned}
& \xi\left(M_{2}, M_{3}\right)=\frac{U^{\prime}\left(M_{3}\right)-U^{\prime}(z)}{M_{3}-z}:=\xi_{0}\left(M_{3}, z\right): \\
& {\left[\omega_{1}(x)+V^{\prime}(x)\right]\left\langle\operatorname{tr} \frac{1}{x-M_{1}} \frac{U^{\prime}\left(M_{3}\right)-U^{\prime}(z)}{M_{3}-z}\right\rangle_{0}+\left\langle\operatorname{tr} \frac{U^{\prime}\left(M_{3}\right)-U^{\prime}(z)}{M_{3}-z} \frac{V^{\prime}\left(M_{1}\right)-V^{\prime}(x)}{x-M_{1}}\right\rangle_{0}} \\
& (4.7)+\left\langle\operatorname{tr} M_{2}^{-1} \frac{1}{x-M_{1}} \frac{U^{\prime}\left(M_{3}\right)-U^{\prime}(z)}{M_{3}-z}\right\rangle_{0}=0 .
\end{aligned}
$$

For the last term in (4.7), we use equation (4.5):

$$
\begin{aligned}
& \left\langle\operatorname{tr} M_{2}^{-1} \frac{1}{x-M_{1}} \frac{U^{\prime}\left(M_{3}\right)-U^{\prime}(z)}{M_{3}-z}\right\rangle_{0} \\
= & \left\langle\operatorname{tr} M_{2} M_{1}^{-1} \frac{1}{x-M_{1}} \frac{U^{\prime}\left(M_{3}\right)-U^{\prime}(z)}{M_{3}-z} M_{3}\right\rangle_{0}+\left(\gamma_{3}-\gamma_{2}\right)\left\langle\operatorname{tr} M_{1}^{-1} \frac{1}{x-M_{1}} \frac{U^{\prime}\left(M_{3}\right)-U^{\prime}(z)}{M_{3}-z}\right\rangle_{0} \\
= & \frac{1}{x}\left\langle\operatorname{tr} M_{2} \frac{1}{x-M_{1}} \frac{U^{\prime}\left(M_{3}\right)-U^{\prime}(z)}{M_{3}-z}\left(M_{3}-z+z\right)\right\rangle_{0}+\frac{1}{x}\left\langle\operatorname{tr} M_{2} M_{1}^{-1} \frac{U^{\prime}\left(M_{3}\right)-U^{\prime}(z)}{M_{3}-z} M_{3}\right\rangle_{0} \\
& +\left(\gamma_{3}-\gamma_{2}\right) \frac{1}{x}\left\langle\operatorname{tr} \frac{1}{x-M_{1}} \frac{U^{\prime}\left(M_{3}\right)-U^{\prime}(z)}{M_{3}-z}\right\rangle_{0}+\left(\gamma_{3}-\gamma_{2}\right) \frac{1}{x}\left\langle\operatorname{tr} M_{1}^{-1} \frac{U^{\prime}\left(M_{3}\right)-U^{\prime}(z)}{M_{3}-z}\right\rangle_{0} \\
= & \frac{z}{x}\left\langle\operatorname{tr} M_{2} \frac{1}{x-M_{1}} \frac{U^{\prime}\left(M_{3}\right)-U^{\prime}(z)}{M_{3}-z}\right\rangle_{0}+\frac{1}{x}\left\langle\operatorname{tr} M_{2} \frac{1}{x-M_{1}}\left(U^{\prime}\left(M_{3}\right)-U^{\prime}(z)\right)\right\rangle_{0} \\
& +\left(\gamma_{3}-\gamma_{2}\right) \frac{1}{x}\left\langle\operatorname{tr} \frac{1}{x-M_{1}} \frac{U^{\prime}\left(M_{3}\right)-U^{\prime}(z)}{M_{3}-z}\right\rangle_{0}+\frac{1}{x}\left\langle\operatorname{tr} M_{2}^{-1} \frac{U^{\prime}\left(M_{3}\right)-U^{\prime}(z)}{M_{3}-z}\right\rangle_{0},
\end{aligned}
$$

where in the last term we have again used substitution (4.5) (in opposite direction). We introduce the polynomials

$$
\begin{aligned}
& P_{n-1, r-1}(x, z):=\left\langle\operatorname{tr} \frac{U^{\prime}\left(M_{3}\right)-U^{\prime}(z)}{M_{3}-z} \frac{V^{\prime}\left(M_{1}\right)-V^{\prime}(x)}{x-M_{1}}\right\rangle_{0}, \\
& Q_{r-1}(z):=\left\langle\operatorname{tr} M_{2}^{-1} \frac{U^{\prime}\left(M_{3}\right)-U^{\prime}(z)}{M_{3}-z}\right\rangle_{0} .
\end{aligned}
$$

Equation (4.7) then becomes

$$
\begin{aligned}
& {\left[\omega_{1}(x)+V^{\prime}(x)+\frac{\gamma_{3}-\gamma_{2}}{x}\right]\left\langle\operatorname{tr} \frac{1}{x-M_{1}} \frac{U^{\prime}\left(M_{3}\right)-U^{\prime}(z)}{M_{3}-z}\right\rangle_{0}} \\
& \quad+\frac{z}{x}\left\langle\operatorname{tr} M_{2} \frac{1}{x-M_{1}} \frac{U^{\prime}\left(M_{3}\right)-U^{\prime}(z)}{M_{3}-z}\right\rangle_{0}+\frac{1}{x}\left\langle\operatorname{tr} M_{2} \frac{1}{x-M_{1}}\left(U^{\prime}\left(M_{3}\right)-U^{\prime}(z)\right)\right\rangle_{0} \\
& \quad+P_{n-1, r-1}(x, z)+\frac{1}{x} Q_{r-1}(z)=0
\end{aligned}
$$

and it remains only to evaluate the term $\left\langle\operatorname{tr} M_{2} \frac{1}{x-M_{1}}\left(U^{\prime}\left(M_{3}\right)-U^{\prime}(z)\right)\right\rangle_{0}$. Note first that, from (4.6), we have that

$$
\left\langle\operatorname{tr} M_{2} \frac{1}{x-M_{1}} U^{\prime}\left(M_{3}\right)\right\rangle_{0}=\left\langle\operatorname{tr} M_{2}^{2} \frac{1}{x-M_{1}}\right\rangle_{0},
$$


and we can evaluate $\left\langle\operatorname{tr} M_{2} \frac{1}{x-M_{1}}\right\rangle_{0}$ and $\left\langle\operatorname{tr} M_{2}^{2} \frac{1}{x-M_{1}}\right\rangle_{0}$ consequently substituting $\xi\left(M_{2}, M_{3}\right)=$ $M_{2}$ and $\xi\left(M_{2}, M_{3}\right)=M_{2}^{2}$ in (4.4). We introduce two more polynomials

$$
\widehat{P}_{n-1}(x):=\left\langle\operatorname{tr} \frac{V^{\prime}\left(M_{1}\right)-V^{\prime}(x)}{x-M_{1}} M_{2}\right\rangle_{0} \text { and } \widehat{\widehat{P}}_{n-1}(x):=\left\langle\operatorname{tr} \frac{V^{\prime}\left(M_{1}\right)-V^{\prime}(x)}{x-M_{1}} M_{2}^{2}\right\rangle_{0} .
$$

The substitution $\xi\left(M_{2}, M_{3}\right)=M_{2}$ results in the equation

$$
\left[\omega_{1}(x)+V^{\prime}(x)\right]\left\langle\operatorname{tr} M_{2} \frac{1}{x-M_{1}}\right\rangle_{0}+\widehat{P}_{n-1}(x)+\omega_{1}(x)=0,
$$

whereas the substitution $\xi\left(M_{2}, M_{3}\right)=M_{2}^{2}$ gives

$$
\left[\omega_{1}(x)+V^{\prime}(x)\right]\left\langle\operatorname{tr} M_{2}^{2} \frac{1}{x-M_{1}}\right\rangle_{0}+\widehat{\widehat{P}}_{n-1}(x)+\left\langle\operatorname{tr} M_{2} \frac{1}{x-M_{1}}\right\rangle_{0}=0,
$$

and we obtain that

$$
\begin{aligned}
& \left\langle\operatorname{tr} M_{2} \frac{1}{x-M_{1}}\right\rangle_{0}=-\frac{\omega_{1}(x)+\widehat{P}_{n-1}(x)}{\omega_{1}(x)+V^{\prime}(x)} \\
& \left\langle\operatorname{tr} M_{2}^{2} \frac{1}{x-M_{1}}\right\rangle_{0}=\frac{1}{\omega_{1}(x)+V^{\prime}(x)}\left[-\widehat{\widehat{P}}_{n-1}(x)+\frac{\omega_{1}(x)+\widehat{P}_{n-1}(x)}{\omega_{1}(x)+V^{\prime}(x)}\right] .
\end{aligned}
$$

Equation (4.10) therefore takes the form

$$
\begin{gathered}
{\left[\omega_{1}(x)+V^{\prime}(x)+\frac{\gamma_{3}-\gamma_{2}}{x}\right]\left\langle\operatorname{tr} \frac{1}{x-M_{1}} \frac{U^{\prime}\left(M_{3}\right)-U^{\prime}(z)}{M_{3}-z}\right\rangle_{0}} \\
+\frac{z}{x}\left\langle\operatorname{tr} M_{2} \frac{1}{x-M_{1}} \frac{U^{\prime}\left(M_{3}\right)-U^{\prime}(z)}{M_{3}-z}\right\rangle_{0}+s(x, z)=0
\end{gathered}
$$

in which $s(x, z)$ is a rational function

$$
\begin{aligned}
& s(x, z)=\quad P_{n-1, r-1}(x, z)+\frac{1}{x} Q_{r-1}(z) \\
& \quad+\frac{1}{x}\left(\frac{1}{\omega_{1}+V^{\prime}(x)}\left[-\widehat{P}_{n-1}(x)+\frac{\omega_{1}(x)+\widehat{P}_{n-1}(x)}{\omega_{1}(x)+V^{\prime}(x)}\right]+U^{\prime}(z) \frac{\omega_{1}(x)+\widehat{P}_{n-1}(x)}{\omega_{1}(x)+V^{\prime}(x)}\right) .
\end{aligned}
$$

Performing the last substitution $\xi\left(M_{2}, M_{3}\right)=\frac{U^{\prime}\left(M_{3}\right)-U^{\prime}(z)}{M_{3}-z} M_{2}$ in (4.4), we obtain

$$
\begin{aligned}
& {\left[\omega_{1}(x)+V^{\prime}(x)\right]\left\langle\operatorname{tr} M_{2} \frac{1}{x-M_{1}} \frac{U^{\prime}\left(M_{3}\right)-U^{\prime}(z)}{M_{3}-z}\right\rangle_{0}} \\
& +\left\langle\operatorname{tr} \frac{1}{x-M_{1}} \frac{U^{\prime}\left(M_{3}\right)-U^{\prime}(z)}{M_{3}-z}\right\rangle_{0}+t(x, z)=0
\end{aligned}
$$

where

$$
t(x, z):=\widehat{P}_{n-1, r-1}(x, z):=\left\langle\operatorname{tr} M_{2} \frac{U^{\prime}\left(M_{3}\right)-U^{\prime}(z)}{M_{3}-z} \frac{V^{\prime}\left(M_{1}\right)-V^{\prime}(x)}{x-M_{1}}\right\rangle_{0}
$$

is again a polynomial function. We now treat Eqs. (4.14) and (4.16) as a system of two linear equations on two unknowns $\left\langle\operatorname{tr} \frac{1}{x-M_{1}} \frac{U^{\prime}\left(M_{3}\right)-U^{\prime}(z)}{M_{3}-z}\right\rangle_{0}$ and $\left\langle\operatorname{tr} M_{2} \frac{1}{x-M_{1}} \frac{U^{\prime}\left(M_{3}\right)-U^{\prime}(z)}{M_{3}-z}\right\rangle_{0}$. We are interested in the case where this system is degenerate, which imposes the constraint on the variable $z$ :

$$
\operatorname{det}\left[\begin{array}{cc}
\omega_{1}(x)+V^{\prime}(x)+\frac{\gamma_{3}-\gamma_{2}}{x} & z / x \\
1 & \omega_{1}(x)+V^{\prime}(x)
\end{array}\right]=0
$$


which gives

$$
z=x\left(\omega_{1}(x)+V^{\prime}(x)\right)\left(\omega_{1}(x)+V^{\prime}(x)+\frac{\gamma_{3}-\gamma_{2}}{x}\right) .
$$

It is a standard trick in multi-matrix models to introduce the new variable $y$ :

$$
y:=\omega_{1}(x)+V^{\prime}(x) .
$$

Then the condition of solvability of the system of linear equations (4.14) and (4.16) is exactly the spectral curve equation

$$
s(x, z)-\left(y+\frac{\gamma_{3}-\gamma_{2}}{x}\right) t(x, z)=0, \quad \text { where } \quad z=x y^{2}+\left(\gamma_{3}-\gamma_{2}\right) y .
$$

Despite its complexity even in the simplest cases (say, we obtain a hyperelliptic curve of maximum genus three for the Gaussian potentials $V(x)$ and $U(z)$ in the Example 4.1 below), we still have an algebraic curve in contrast to the case of Hurwitz numbers in the case of branching points with only simple ramifications for which it was conjectured in [7] and shown in [6] that the corresponding spectral curve in the case of simple Hurwitz numbers is the Lambert curve given by a nonpolynomial equation $x=y e^{-y}$.

Example 4.1. Let us consider the case of Gaussian potentials $V(x)=x^{2} / 2$ and $U(z)=z^{2} / 2$. Then all the polynomials $P_{n-1, r-1}, \widehat{P}_{n-1, r-1}, \widehat{P}_{n-1}, \widehat{\widehat{P}}_{n-1}$, and $Q_{r-1}$ are constants and, moreover, $P_{n-1, r-1}=1$ and $\widehat{P}_{n-1, r-1}=\widehat{P}_{n-1}$. Then, after all cancelations, we obtain the spectral curve equation

$$
y-x+\widehat{P}-\widehat{\widehat{P}} y+x y^{2}+Q y^{2}+y^{2}(y-x)\left(x y+\gamma_{2}-\gamma_{3}\right)=0,
$$

which, for the general values of constants in (4.22), describes a hyperelliptic curve of genus three.

\section{Conclusion}

We have constructed the chain of matrix representation for the generating functions for numbers of generalized Belyi fat graphs for hypergeometric Hurwitz numbers with ramifications at $n$ distinct points and with ramification profiles fixed at two of these $n$ points. We also distinguish between fat graphs with different numbers of pre-images of other ramification points. The corresponding partition functions lie in the generalized Kontsevich matrix-model class thus being tau functions of the KP hierarchy, which was previously shown from the character expansion standpoint in 21. We were able to construct the chain of matrix representation with a nonstandard interaction $\sum_{i=3}^{n} \operatorname{tr}\left(M_{i-1} M_{i}^{-1}\right)$ between neighbor Hermitian positive-definite matrices in the chain in the case where we do not distinguish between variables of $n-3$ cycles. We were successful in proposing a method for solving models with interactions of this sort. For the simplicity sake, in this note we have restricted our consideration to the model case of the two-dimensional Toda chain hierarchy with one intermediate matrix (the case $n=4$ ), but our method can be straightforwardly generalized to the case of $n-3$ intermediate matrices with the last, $n$th matrix, being an external field $|\Lambda|^{-2}$.

It is interesting to establish other relations. For instance, generating function (1.2) in the case of clean Belyi morphisms is related [3] to the free energy of the Kontsevich-Penner matrix model [12], [13], which is known (see [9], [26], [14]) to be the generating function of the numbers of integer points in moduli spaces $\mathcal{M}_{g, s}$ of curves of genus $g$ with $s$ holes with fixed (integer) perimeters; the very same model was also related [9] by a canonical transformation to two copies of the Kontsevich matrix model expressed in times related to the discretization of the moduli spaces $\mathcal{M}_{g, s}$. It is tempting to generalize these discretization patterns to cut-and-join 
operators of [30] and [2] in the case of hypergeometric Hurwitz numbers and to Hodge integrals of [22].

\section{ACKNOWLEDGMENTS}

it is our pleasure to celebrate with this paper the 75th birthday of Andrey Alekseevich Slavnov, an outstanding scientist, a great personality, and the teacher of one of us (L.Ch.).

The authors acknowledge support from the ERC Advance Grant 291092 "Exploring the Quantum Universe" (EQU). J.A. acknowledges support of the FNU, the Free Danish Research Council, from the grant "Quantum gravity and the role of black holes." The work of L.Ch. was supported by the Russian Foundation for Basic Research (Grant Nos. 14-01-00860-a and 1301-12405-ofi-m) and by the Program 19-P of the Russian Academy of Sciences "Fundamental Problems of Nonlinear Dynamics."

\section{REFERENCES}

[1] A. Alexandrov, A. Mironov and A. Morozov, Partition functions of matrix models as the first special functions of String Theory I. Finite size Hermitean 1-matrix model, Int. J. Mod. Phys. A19 (2004) 41274165 .

[2] A. Alexandrov, A. Mironov, A. Morozov, and S. Natanzon, On KP-integrable Hurwitz functions, arXiv:1405.1395

[3] J. Ambjørn and L. Chekhov, The matrix model for dessins d'enfants, Ann. Inst. Henri Poincaré, Comb. Phys. Interact. 1 (2014) 337-361; DOI 10.4171/AIHPD/10.

[4] J. Ambjørn, C. F. Kristjansen, and Y. M. Makeenko, Higher genus correlators for the complex matrix model, Mod. Phys. Lett. A7 (1992) 3187-3203; hep-th/9207020.

[5] G. Belyi, On Galois extension of a maximal cyclotomic field, USSR Math. Izvestiya 14:2 (1980) 247-256

[6] G. Borot, B. Eynard, M. Mulase, and B. Safnuk, A matrix model for simple Hurwitz numbers, and topological recursion, J. Geom. Phys. 61(2) (2011) 522-540; arXiv:0906.1206.

[7] V. Bouchard and M. Mariño, Hurwitz numbers, matrix models, and enumerative geometry, in: From Hodge Theory to Integrability and tQFT: tt*-geometry, Proceedings of Symposia in Pure Mathematics, AMS (2008); arXiv:0709.1458

[8] L.Chekhov, Matrix models with hard walls: Geometry and solutions, J. Phys. A 39 (2006) 8857-8894; hep-th/0602013.

[9] L. Chekhov, Matrix models tools and geometry of moduli spaces, Acta Appl. Mathematicae 48 (1997) 33-90; e-Print Archive: hep-th/9509001.

[10] L. Chekhov, B. Eynard, Hermitean matrix model free energy: Feynman graph technique for all genera, JHEP 0603:014 (2006); hep-th/0504116.

[11] L. Chekhov, B. Eynard, and N. Orantin, Free energy topological expansion for the 2-matrix model, JHEP 12(2006)053; hep-th/0603003.

[12] L. Chekhov and Yu. Makeenko, The multicritical Kontsevich-Penner model, Mod. Phys. Lett. A7 (1992) 1223-1236; hep-th/9201033.

[13] L. Chekhov and Yu. Makeenko, A hint on the external field problem for matrix models, Phys. Lett. B278 (1992) 271-278; hep-th/9202006

[14] Norman Do and Paul Norbury, Pruned Hurwitz numbers, arXiv:1312.7561v1.

[15] O. Dumitrescu, M. Mulase, B. Safnuk, and A. Sorkin, The spectral curve of the Eynard-Orantin recursion via the Laplace transform, arXiv:1202.1159.

[16] B. Eynard, All genus correlation functions for the hermitian 1-matrix model, JHEP 0411:031 (2004).

[17] B. Eynard and A. Prats Ferrer, Topological expansion of the chain of matrices, JHEP 0907 (2009) 096; arXiv:0805.1368v2.

[18] I. P. Goulden and D. M. Jackson, The KP hierarchy, branched covers, and triangulations, arXiv:0803.3980.

[19] A. Grothendieck, Esquisse d'un programme, Geometric Galois Action, Cambridge Univ. Press, Cambridge (1997) 5-48.

[20] M. Guay-Paquet and J. Harnad, Generating functions for weighted Hurwitz numbers, arXiv:1408.6766.

[21] J. Harnad and A. Yu. Orlov, Hypergeometric $\tau$-functions, Hurwitz numbers and enumeration of paths, arXiv:1407.7800

[22] M. Kazarian, KP hierarchy for Hodge integrals, Adv. Math. 221 (2009) 1-21. 
[23] M. Kazarian and P. Zograf, Virasoro constraints and topological recursion for Grothendieck?s dessin counting, arXiv:1406.5976.

[24] S. Kharchev, A.Marshakov, A.Mironov, A.Morozov, and A. Zabrodin, Unification of all string models with $c<1$, Phys.Lett., 275B (1992) 311-314.

S. Kharchev, A.Marshakov, A.Mironov, A.Morozov, and A. Zabrodin, Towards unified theory of 2d gravity, Nucl. Phys., B380 (1992) 181-240.

[25] A. Mironov, A. Morozov, and G. Semenoff, Unitary matrix integrals in the framework of Generalized Kontsevich Model. I. Brezín-Gross-Witten model, Int. J. Mod. Phys. A10 (1995) 2015-2040.

[26] P. Norbury, Counting lattice points in the moduli space of curves, Math. Res. Lett. 17 (2010) 467-481.

[27] A. Okounkov and R. Pandharipande, Gromov-Witten theory, Hurwitz numbers, and completed cycles, Ann. Math. 163 (2006) 517-590; math.AG/0204305.

[28] A. Orlov and D. M. Shcherbin, Hypergeometric solutions of soliton equations, Theor. Math. Phys. 128 (2001) 906-926

[29] A. Orlov, Hypergeometric functions as infinite-soliton tau functions, Theor. Math. Phys. 146 (2006) 183206.

[30] P. G. Zograf, Enumeration of Grothendieck's dessins and KP hierarchy, arXiv:1312:2538v2. 